\title{
THE GALACTIC DISC AGE-METALLICITY RELATION
}

\author{
Y.K. NG \\ Padova Astronomical Observatory \\ AND \\ G. BERTELLI, G. CARRARO, L. PORTINARI \\ Padova Department of Astronomy \\ Vicolo dell'Osservatorio 5, I-35122 Padova, ITALY
}

\begin{abstract}
Ng} \&$ Bertelli (1998) computed new ages for the stars from the Edvardsson et al. (1993) data set. First the effects due to a change of isochrones were studied. Then the ages were derived using the Hipparcos parallaxes. The large spread in metallicity at any particular age is likely intrinsic to the disc and not due to the overlap of other galactic components. The resulting age-metallicity relation (AMR) is compared with those obtained from other methods, i.e. galactic open clusters, stellar population synthesis (star counts), and chemical evolution models. Details about the comparison of the various AMRs can be found in Carraro et al. (1997). The AMR for open clusters is corrected for the present day radial gradient: $-0.07 \mathrm{dex} \mathrm{kpc}^{-1}$ (unweighted least-squares for clusters younger than $2 \mathrm{Gyr}$ ); within the uncertainties the average correction is independent of age. No correction for the vertical gradient was applied, since it is likely due to insufficient discrimination between age groups. The AMRs of open clusters and stars show a similar trend in the scatter and might provide clues about infalling and/or merger events.
\end{abstract}

Acknowledgements of support received from the Italian Space Agency (ASI), the Italian Ministry of University and Scientific \& Technological Research (MURST), the TMR network of the EC (grant ERBFMRX-CT96-0086), and the Italian National Council of Research (CNR-GNA).

\section{References}

Carraro G., Ng, Y.K., Portinari L., 1997, MNRAS submitted (astro-ph/9707185)

Edvardsson B., Andersen J., Gustafsson B., et al., 1993, A\&A 275, 101

Ng Y.K., Bertelli G., 1998, A\&A in press (astro-ph/9707043) 